\title{
Smoking Burden in the Philippines
}

\author{
Felix Eduardo R. Punzalan, ${ }^{1}$ Paul Ferdinand M. Reganit, ${ }^{1,2}$ Eugene B. Reyes ${ }^{1}$ \\ and The National Nutrition and Health Examination Survey (NNHES) Group \\ ${ }^{1}$ Section of Cardiology, Department of Medicine, College of Medicine and Philippine General Hospital, University of the Philippines Manila \\ ${ }^{2}$ Department of Epidemiology and Biostatistics, College of Public Health, University of the Philippines Manila
}

\begin{abstract}
Background. The $2^{\text {nd }}$ National Nutrition and Health Survey (NNHeS II) was conducted in 2008 to measure the prevalence of lifestyle-related diseases and risk factors among adult Filipinos 20 years or older. One of the risk factors measured was smoking.
\end{abstract}

Methods. The survey used a stratified multi-stage sampling design and covered the 17 regions of the country. The clinical component included Filipino adults 20 years or older. Data was collected using health questionnaires; anthropometric measurements were made, and blood examinations performed on all participants. The prevalence of smoking was determined.

Results. A total of 7,700 subjects were included in the survey. The over-all prevalence of current smokers was $31 \%(53.2 \%$ and $12.5 \%$ for men and women, respectively). $14 \%$ were former smokers ( $20.9 \%$ of men and $9.5 \%$ of women) while $25.9 \%$ of men and $78.1 \%$ of women were never smokers.

Among current male smokers, the highest prevalence $(57.7 \%)$ belonged to the 20-29 age group. While among current female smokers, the highest prevalence (23.2\%) belonged to the greater than 70-year-old category.

There were more current smokers in the rural areas (33.1\%) than the urban areas (28.9\%) and there were more current and former smokers among respondents whose educational attainment was at the elementary level ( $35.1 \%$ and $16.4 \%$, respectively). While there were more never smokers whose educational attainment was at least the tertiary level (62.7\%).

Conclusion. Tobacco use in the Philippines is still prevalent at $31 \%$ and is predominantly composed of males in the 20-29 age group. Current smoking is highest among rural dwellers and among individuals with elementary education, while majority of women and college level respondents are never smokers.

Key Words: tobacco use, tobacco burden, smoking, Philippines, tobacco prevalence

\footnotetext{
Corresponding author: Paul Ferdinand M. Reganit, MD, MPH

Section of Cardiology

Department of Medicine

Philippine General Hospital

University of the Philippines Manila

Taft Avenue, Ermita, Manila 1000 Philippines

Telephone: +6325260784 local 132

Email: preganit@post.harvard.edu / preganit@netscape.net
}

\section{Introduction}

\section{Epidemiology}

Smoking is the most common cause of preventable death in the world causing five million deaths per year and is projected to increase to 8 million per year by 2030; three quarters of these deaths will be in low- and middle-income countries according to the World Health Organization. ${ }^{1,2}$ The Western Pacific Region of which the Philippines is part has the highest prevalence of male smoking including the highest level of uptake of smoking by women and young people. $2,3,4$

The World Health Organization through the Tobacco Free Initiative (WHO-TFI) is assisting governments in creating national tobacco disease control and prevention programs and strategies aimed at stemming the tide of the global tobacco epidemic. Specifically through the MPOWER package which is a series of six proven policies which aim to (i) Monitor tobacco use and prevention policies; (ii) Protect people from tobacco smoke; (iii) Offer help to quit tobacco use; (iv) Warn about the dangers of tobacco; (v) Enforce bans on tobacco advertising, promotion, and sponsorship; and (vi) Raise taxes on tobacco. ${ }^{3,5}$

\section{Prior Studies}

In 1999, the $5^{\text {th }}$ Food and Nutrition Research Institute National Nutrition Survey showed the following results:

The over-all prevalence of current smokers based on this survey was $33 \%(53.79 \%$ and $11.07 \%$ for men and women, respectively. $12.79 \%$ were former smokers $(20.87 \%$ of men and $5.18 \%$ of women) while $25.15 \%$ of men and $82.20 \%$ of women were never smokers.

Among current male smokers, the highest prevalence (56.73\%) belonged to the $40-49$ age category, followed by the 20-29 age group (56.23\%). It is lowest among respondents older than 60 years of age. Among current female smokers, the highest prevalence (17.67\%) belonged to the 40-59 age category, followed by the older than 60 age group (16.87\%). It was lowest among the respondents in the 20-29 age group.

The highest prevalence of former male smokers belonged to the older than 60 years category $(40.58 \%)$, followed by the $40-59$ age group $(24.87 \%)$. It was lowest in the 20-39 age group (29.89\%). In like manner, the highest prevalence of former female smokers belonged to the older 
than 60 years category (11\%), followed by the 40-59 age group $(5.32 \%)$. It was lowest in the $30-29$ age group $(3.91 \%)$.

In 2003, the $2^{\text {nd }}$ National Nutrition and Health Examination Survey showed the following results ${ }^{6,7}$ :

The over-all prevalence of current smokers based on this survey was $34.8 \%(56.3 \%$ and $12.1 \%$ for men and women, respectively. $15.1 \%$ of men and $5.1 \%$ of women were former smokers.

\section{Recent Studies}

In 2009, the Philippines participated in the Global Adult Tobacco Survey (GATS) through the Department of Health $(\mathrm{DOH})$ and the National Statistical Office (NSO) in collaboration with the World Health Organization and the United States Centers for Disease Control and Prevention (CDC). ${ }^{4,5,8}$

The 2009 GATS (Philippines) was a nationally representative household survey of adult Filipinos older than 15 years. It collected data on tobacco use and control measures. The study showed the following results: “(i) the overall prevalence of current tobacco use was $28.3 \%$ of 17.3 million population older than 15 years old; $47.7 \%(14.6$ million) men, and 9.0\% (2.8 million) women, (ii) eighty percent of current smokers are daily smokers; (iii) manufactured cigarettes is the type of product smoked by $98 \%$ of men and $83 \%$ of women smokers."

In addition, according to the study, among daily cigarette smokers, on average, men smoke 11.3 cigarettes and women 7.0 cigarettes per day. The mean or average age of initiation of smoking among daily smokers age 18-34 years was 17.4 years for men and 19.1 years for women. Overall, $2.8 \%$ of men and $1.2 \%$ of women currently use smokeless tobacco.

Moreover, the same study showed that "overall, 94.0\% believe that smoking causes serious illness. But the belief that smoking causes specific illness varies: stroke $(75.5 \%)$, heart attack $(81.3 \%)$, and lung cancer $(95.6 \%)^{\prime \prime} .4$

Prior studies on adult smoking in the Philippines were conducted by the Department of Health and the Lung Center of the Philippines (LCP) in 1989. ${ }^{9}$ Among subjects older than 18 years, the prevalence of current smokers was $58.6 \%{ }^{9}$ However, a more recent study conducted by the Social Weather Station in 2009 showed an overall prevalence of current smoking at 27\% (SWS 2009) which closely approximated the 2009 Philippines' GATS overall prevalence of current smoking at $31 \%{ }^{4}$

The prevalence of teenage smoking is also significant in the Philippines. The Global Youth Tobacco Survey (GYTS) conducted in the Philippines in 2007 among subjects aged 13 to 15 years old showed the following results: "17.5\% of the students currently smoked cigarettes and $7.7 \%$ currently used other tobacco products. Boys (23.4\%) were more likely than girls $(12.0 \%)$ to currently smoke cigarettes; however, there was no gender difference in the use of other tobacco products $\left(8.2 \%\right.$ and $7.2 \%$, respectively) ${ }^{\prime \prime} .8$

\section{Impact}

The economic cost attributable to smoking in terms of the four smoking-related diseases (lung cancer, cardiovascular diseases, coronary artery disease, and chronic obstructive lung disease) is considerable. In the Tobacco and Poverty Study in the Philippines, "total costs of illness for the four smoking-related diseases studied were estimated at USD 6.05 billion using the Smoking Attributable Morbidity and Mortality and Economic Costs (SAMMEC) figures, while Peto-Lopez estimates yielded a more conservative but still substantial loss of USD 2.86 Billion". 4,8

The health, social, economic, and public health burden of tobacco use is staggering and all sectors are enjoined in stemming the tide of the global tobacco epidemic.

\section{Objectives}

\section{General Objective:}

To determine the prevalence of smoking in the Philippines using the 2008 National Nutrition and Health Examination Survey Report

Secondary Objectives:

1. To determine the prevalence of smoking according to (i) gender, (ii) age, (iii) rural or urban residence, and (iv) educational attainment

3. To compare the prevalence of smoking by gender with the 1999 and 2003 National Nutrition and Health Survey (NNHeS)

\section{Methods}

The survey utilized stratified multi-stage sampling design to represent each of the 17 regions in the country. The detailed methodology of this survey has been described by the Food and Nutrition Research Institute. ${ }^{10}$

The clinical component which surveyed the prevalence of atherosclerosis-related risk factors (hypertension, diabetes, dyslipidemia, smoking and obesity) and diseases (coronary, cerebrovascular and peripheral artery disease) included adult subjects 20 years and over. Tobacco use was determined.

The study used standardized methods of anthropometric, clinical, and health assessment for the data collection. Statistical analyses included mean, median, and standard deviation of quantitative variables; and proportions for qualitative data.

\section{Definition of Terms}

1. Smoking status is based on the three World Health Organization categories: 
1. Never- smoker for those who have never tried smoking tobacco or cigarette

2. Former-smoker for those who have tried but did not continue or are no longer using tobacco or cigarette

3. Current-smoker for those who were still smoking tobacco or cigarettes during the survey day.

2. Urban residence:

According to the National Statistics Office (NSO), an area is considered urban if it is a:

1. City or municipality having a population density of at least 1,000 persons per square kilometer

2. Central district of municipalities and cities which have a population density of at least 500 persons per square kilometer

3. Poblacion or central district which has the following:

a. At least six commercial establishments

b. At least three of the following ( a town hall, church or chapel with religious services of at least once a month, a public plaza, park, or cemetery, and a market place where trading activities are carried on at least once a week, and a public building like school, hospital, puericulture, and health center or library).

c. Barangay having at least 1000 inhabitants which meet the conditions set in number 3 above, and where the occupation of the inhabitants is predominantly non-farming or non-fishing.

d. All areas not falling under any of the above classifications are considered Rural (NSO 2004)

3. Rural residence: Not urban

4. Educational attainment:

(i) Elementary (Grade 1-6)

(ii) Secondary (High School Year 1-4)

(iii) At least tertiary (Post Secondary Year 1)

\section{Results}

The Clinical Component of the 2008 National Nutrition and Health Survey included 3,744 households from 79 provinces with 7700 eligible subjects.

Over-all Prevalence of Smoking According to Gender and Age

The over-all prevalence of current smokers based on this survey was $31 \%(53.2 \%$ and $12.5 \%$ for men and women, respectively (Table 1). $14 \%$ were former smokers $(20.9 \%$ of men and $9.5 \%$ of women) while $25.9 \%$ of men and $78.1 \%$ of women were never smokers.

Among current male smokers, the highest prevalence $(57.7 \%)$ belonged to the $20-29$ age category, followed by the $40-49$ age group $(55.5 \%)$. It is lowest among respondents older than 70 years of age.
Among current female smokers, the highest prevalence (23.2\%) belonged to the older than 70 years category, followed by the 50-59 age group (15.3\%). It was lowest among the respondents in the 20-29 age group.

The highest prevalence of former male smokers belonged to the older than 70 years category $(39.5 \%)$, followed by the 60-69 age group (34.7\%). It was lowest in the 20-29 age group $(11.3 \%)$. In like manner, the highest prevalence of former female smokers belonged to the older than 70 years category $(20.1 \%)$, followed by the $60-69$ age group (13.1\%). It was lowest in the $30-39$ age group (5.8\%).

Table 1. Prevalence of Never, Current and Former Tobacco Use, by Age and Gender. 2008 National Nutrition and Health Survey. Philippines.

\begin{tabular}{cccc}
\hline \multirow{2}{*}{ Gender/ Age } & \multicolumn{3}{c}{ Level of Tobacco Use } \\
\cline { 2 - 4 } & $\begin{array}{c}\text { Never } \\
\text { \% (S.E) }\end{array}$ & $\begin{array}{c}\text { Current } \\
\text { \% (S.E) }\end{array}$ & $\begin{array}{c}\text { Former } \\
\text { \% (S.E) }\end{array}$ \\
\hline Male & $\mathbf{2 5 . 9 ( 0 . 8 )}$ & $\mathbf{5 3 . 2}(\mathbf{0 . 9 )}$ & $\mathbf{2 0 . 9}(\mathbf{0 . 8 )}$ \\
$20-29$ & $31.0(1.8)$ & $57.7(1.9)$ & $11.3(1.2)$ \\
$30-39$ & $29.6(1.7)$ & $53.2(1.9)$ & $17.2(1.6)$ \\
$40-49$ & $23.9(1.6)$ & $55.5(1.9)$ & $20.6(1.6)$ \\
$50-59$ & $17.6(1.8)$ & $54.6(2.4)$ & $27.8(2.2)$ \\
$60-69$ & $20.4(2.4)$ & $45.0(3.0)$ & $34.7(2.9)$ \\
$\geq 70$ & $27.6(3.4)$ & $32.9(3.5)$ & $39.5(3.8)$ \\
Subtotal & & & \\
Female & $\mathbf{7 8 . 1 ( 0 . 8 )}$ & $\mathbf{1 2 . 5 ( 0 . 6 )}$ & $\mathbf{9 . 5 ( 0 . 5 )}$ \\
$20-29$ & $81.5(1.5)$ & $8.6(1.0)$ & $10.0(1.2)$ \\
$30-39$ & $83.4(1.4)$ & $10.8(1.1)$ & $5.8(0.9)$ \\
$40-49$ & $79.8(1.6)$ & $12.1(1.3)$ & $8.1(1.0)$ \\
$50-59$ & $75.6(1.7)$ & $15.3(1.5)$ & $9.1(1.2)$ \\
$60-69$ & $72.1(2.4)$ & $14.8(1.8)$ & $13.1(1.8)$ \\
$\geq 70$ & $56.6(3.1)$ & $23.2(2.6)$ & $20.1(2.7)$ \\
Subtotal & & & $\mathbf{1 4 . 7 ( 0 . 5 )}$ \\
\hline Overall & $\mathbf{5 4 . 3 ( 0 . 6 )}$ & $\mathbf{3 1 . 0 ( 0 . 6 )}$ &
\end{tabular}

Prevalence of Smoking According to Residence

The prevalence of current smokers was $33.1 \%$ and $28.9 \%$ in the rural and urban areas, respectively. While the prevalence of former smokers was $14.6 \%$ and $14.9 \%$ in the rural and urban areas, respectively (Table 2).

Table 2. Prevalence of Never, Current and Former Tobacco Use by Residence, 2008 National Nutrition and Health Survey. Philippines.

\begin{tabular}{lccc}
\hline & \multicolumn{3}{c}{ Level of Tobacco Use } \\
\cline { 2 - 4 } & Never & Current & Former \\
Residence & $\%$ (S.E) & \% (S.E) & $14.6(0.66)$ \\
\hline Rural & $52.3(0.84)$ & $33.1(0.79)$ & $14.9(0.74)$ \\
Urban & $56.2(0.98)$ & $28.9(0.86)$ & (S)
\end{tabular}

Prevalence of Smoking According to Educational Attainment

There were more current and former smokers among those whose educational attainment was at the elementary level, $35.1 \%$ and $16.4 \%$, respectively. While the prevalence of never smoker was highest among those who have at least tertiary education $(62.7 \%)$.(Table 3 ) 
Table 3. Prevalence of Never, Current and Former Smoking by Educational Attainment, 2008 National Nutrition and Health Survey. Philippines.

\begin{tabular}{lccc}
\hline & \multicolumn{3}{c}{ Level of Tobacco Use } \\
\cline { 2 - 4 } Educational Attainment & Never & Current & Former \\
\hline Elementary & $48.5(1.1)$ & $35.1(1.0)$ & $16.4(0.81)$ \\
Secondary & $54.2(1.0)$ & $31.6(0.91)$ & $14.2(0.78)$ \\
At least tertiary & $62.7(1.2)$ & $23.7(1.0)$ & $13.6(0.88)$ \\
\hline
\end{tabular}

Comparison of Prevalence of Current Smokers using the 1999 FNRI NNS, 2003 NNHeS, and 2008 NNHeS Surveys

Similar national surveys were conducted in 1999 and 2003.7,10 The smoking prevalence was reported in all the surveys (Table 4). In 1999, the overall prevalence of current smoking was $31.61 \%$ compared to $34.8 \%$ in 2003 . The 2008 survey showed a lower prevalence of $31.0 \%$.

All surveys showed that more than half of the male respondents were current smokers $(53.79 \%, 56.3 \%$ and $53.2 \%$ ). However, female current smokers increased from $11.07 \%$ to $12.1 \%$ and $12.5 \%$ in the 2008 NNHeS Survey.

Table 4. Comparison of Prevalence of Overall Current Tobacco Use by Gender Using the 1999 FNRI NNS, 2003 NNHeS and 2008 NNHeS Surveys. Philippines

\begin{tabular}{lccc}
\hline \multicolumn{3}{c}{ Current Tobacco Users } \\
\hline & 1999 & 2003 & 2008 \\
Overall & $31.61 \%$ & $34.8 \%$ & $31 \%$ \\
Male & $53.79 \%$ & $56.3 \%$ & $53.2 \%$ \\
Female & $11.07 \%$ & $12.1 \%$ & $12.5 \%$ \\
\hline
\end{tabular}

\section{Discussion}

The World Health Organization has staged tobacco use as follows: Stage $1-$ low $(<20 \%)$, male and minimal female prevalence; Stage 2 - high $(>50 \%)$, male and rising female prevalence; Stage 3 - sharp declines among men, gradual declines in women; and Stage 4 - further declines in both; peaks in tobacco-related deaths. ${ }^{1,2}$

Tobacco use typically starts among the wealthy; however it expands to the low to middle income populations of both sexes. Smoking is not only a public health concern among developed economies but in developing countries as well where adult tobacco use is on the rise among teenagers, women and young adults. $3,4,5,8$

In the Philippines, about one out of 3 adults are current smokers and are predominantly young men in the 20-29 years age group with complex socio-cultural-psychological dynamics influencing and sustaining its use. Women current smokers are still in the minority maybe due to the interplay of societal pressure, role expectations, acceptable behavior, and current mores. ${ }^{3,4,5,8}$

More idle time and lack of employment maybe the postulated reasons for more current smokers in the rural areas while the lack of knowledge regarding the possible adverse effects of tobacco on health maybe the reason for the higher prevalence of current smokers among individuals with elementary education. ${ }^{3,4,5,8}$

A higher proportion of individuals with at least tertiary education comprise the never smoker group. Intuitively, a college level education confers a personal awareness and appreciation of the deleterious effects of tobacco use. . $^{3,4,5}$,

The current data showed that the overall prevalence of current smokers in the Philippines has fluctuated from $31.61 \%$ to $34.5 \%$ and eventually $31 \%$ using the 1999 FNRI-NNS, 2003 NNHeS, and 2008 NNHeS reports, respectively. $6,7,10$

Male current smokers showed a similar pattern from $53.79 \%$ to $56.3 \%$ and eventually $53.2 \%$ using the 1999 FNRINNS, 2003 NNHeS, and 2008 NNHeS reports, respectively. However, female current smokers showed an increasing trend from $11.07 \%$ to $12.1 \%$ and eventually $12.5 \%$ using the same survey reports. This reflects increasing intake of smoking among women..$^{3,4,8}$

\section{Conclusion}

Tobacco use in the Philippines is still prevalent at 31\% and is predominantly composed of males in the 20-29 age group. Current smoking is highest among rural dwellers and among individuals with elementary education, while majority of women and college level respondents are never smokers.

\section{Acknowledgments}

We would like to acknowledge all the members of the National Nutrition and Health Examination Survey Group.

\section{References}

1. Esson K. The Millenium Development Goals and Tobacco Control. United Nations Development Group. 2006

2. World Health Organization. Global Adult Tobacco Survey. 2003

3. WHO. Regional Action Plan for the Tobacco Free Initiative in the Western Pacific Region. 2010-2014

4. WHO. Philippine Country Report (Global Adult Tobacco Survey). 2009 5. Tobacco Free Center. Philippine Tobacco Burden Facts. 2003

6. Food and Nutrition Center and Department of Science and Technology. 2008 National Nutrition and Health Survey. Philippines. 2008

7. Dans AL, Morales DD, Velandria FV, et al for the NNHeS: 2003 Group. National Nutrition and Health Survey (NNHeS): AtherosclerosisRelated Diseases and Risk Factors. Philip J Intern Med. 2005; 43:103-15.

8. Tobacco and Poverty Study in the Philippines. Philippines Global Adult Tobacco Survey (GATS) Report 200. March 2010

9. Baquilod MM. Review of previous national surveys on prevalence of cigarette smoking in the Philippines 1989-2009.

10. Sy RG, Dans AL, Punzalan FER, Amarillo ML, Velandria F for the FNRI-HDL Study Group. The prevalence of dyslipidemia, diabetes, hypertension, stroke and angina pectoris in the Philippines. Philip J Intern Med. 2003; 41:1-6. 